\title{
Exigency of Vehicular Accidents Detection through Paging using GSM Technology
}

\begin{abstract}
Malla Poojith
Department of Electronics and Communication Engineering. Amrita School Of Engineering, Bengaluru, Amrita Vishwa Vidyapeetham, India.
\end{abstract}

poojith8@gmail.com

\begin{abstract}
Nowadays, the rate of vehicular accidents is increasing at a tremendous rate. There are many causes of road accidents and fatalities. One of the important causes of the increasing fatality rate is the lack of immediate medical attention. This problem can be solved if immediate medical attention can be provided to the accident victims. There are a lot of existing algorithms that were designed for this purpose. In this paper, an algorithm is proposed, which utilizes cellular communication paging service to notify regarding the accidents. This process is mobile device independent as this setup is fixed to the car and uses the GSM module for requesting medical emergency.
\end{abstract}

Keywords: Road accidents, Fatality Rate, Cellular communication, paging service, Mobile device, GSM module, medical emergency

\section{Introduction}

Transportation has become one of the important parts of our daily life irrespective of the age group. The population is increasing drastically in every part of the world. As the population is increasing the demand for transportation is also increasing proportionately. As a result, the automotive industries are have shown tremendous enhancement in its production over recent years. Millions of vehicles are manufactured every year. Proportionately the vehicular accidents are also increasing day-by-day due to this increase in the vehicular population. According to a study in 2018 [1], in India 16 citizens are killed every hour due to road accidents. Most importantly, $40 \%$ of the die due to the lack of immediate medical attention. In India, most of the accidents occur in highways where the hospitals are not available in the nearby vicinities [2].

Most of the accidents occur due to the driver's negligence such as rash driving and the driver's health condition and also lack of proper infrastructure. In any case, immediate medical attention would reduce the accidents rate. In such cases a fraction of second would greatly matter in some cases it might cost the victims life. To deal with this problem, an automatic notification can be given to the nearest emergency services and also to the nearest available doctors in that vicinity providing them with the details like the location and the car details so that tracking would be easier.
In this paper, an algorithm is proposed for an SOS emergency case where the driver can request for an emergency in case of medical emergencies and also an automatic emergency can be triggered in case of accidents and the deployment of airbags in the car. The triggered alarm not only notifies the nearest emergency services but also notifies the nearest medical personnel available.

In this paper, Section 2 describes the related works, Section 3 describes the block diagram and proposed algorithm, and Section 4 shows the flowchart of the proposed algorithm for a better understanding of the solution and Section 5 gives the conclusion of the proposed model.

\section{Related Work}

A unique algorithm is designed [3] to notify regarding the accidents to the medical authorities. They considered speed as the criteria in developing the algorithm. They used GPS module to monitor the speed and considered threshold speeds for different cases and of the actual speed was less than the threshold speed, then an alarm was raised for the accident and the location, the speed and the time were then sent using the GPRS modem and a voice call is established to know further details if possible. This way the accidents were notified. But considering the speed to be the only reason for the accident is not appropriate in all the cases. There might be some cases in which the stationary cars might also get struck by the other moving vehicles.

In this paper [4] they used RF antenna and GPRS modem to send the accidents data. They considered impact sensor input to trigger the accident alarm and then the data is transmitted to the service provider end, where the service provider has to then notify these details to the ambulance and police services. In this, they used an RF antenna to transmit on the transmission side and RF receiver on the receiver side to receive the data. The data is sent in a message code which is then decoded at the receiver's end. One of the main drawbacks in this process is the RF module, which has a very limited range of communication. This range gets affected depending on the climatic conditions and also RF works best only in Line of Sight (LoS) propagation, which keeps this algorithm at a disadvantage.

The base concept used in this paper [5], was the Internet of Things (IoT). They used NFC to identify the driver details 
and they considered the Public Sector Organization (PSO) HQ as their base contact. They used a mobile application to monitor and interface all the user data. This application has a database of all the users, where the user's health data and blood group data are stored and retrieved as soon the accident occurs. The user is identified using the NFC tap of their mobile to the car and as soon as the accident occurs, this data is sent immediately to the HQ. The accident is identified by shock sensors that are attached to the car and use cellular IoT to convey this to the PSO HQ. This data includes the passenger's health data and blood group along with the location of the accident. The PSO HQ then shares this data to the PSO rescue team that is allocated to that area, who can come for their rescue. The main disadvantage of this model us that, the complete interaction occurs through the mobile device. None of this information can be transmitted if the mobile device is dead or damaged during the accident or if the mobile is not present in the car. In that case, this fails to notify this to the organization.

This paper [6] is a similar concept to that of [5], that is a mobile application based. But, uses completely different working compared to the previous one. In this paper, they use an app for interface and acquire required data from pressure sensors, GPS and accelerometer. The pressure sensors are used to sense the collision, GPS is used to monitor the speed of the vehicle and the accelerometer check the angle of the vehicle. Every parameter is set with a threshold value and compared with the actual vehicles to detect the occurrence of accidents. Then, if an accident is detected, this location data is sent to the nearest hospital and police station as an emergency message alert and this data is also sent to the emergency contact set by the driver. As mentioned previously, the major drawback of smart phonebased method is the availability of the smartphone in the car and also the working condition of the phone after the impact or after the accident.

This paper [7] uses a completely different approach compared to the previous algorithms. This algorithm uses Vehicle to vehicle (V2V) communication and Vehicle to Infrastructure (V2I) communication to notify regarding the accident to the emergency services. The main device used here is the OBU unit, which is installed to the car. Firstly, it tries to communicate through V2I communication, where if an accident occurs, it directly checks to establish communication from the vehicle to the nearest cell tower. If the cell tower is too far from the vehicle, then $\mathrm{V} 2 \mathrm{~V}$ communication is done and finally, V2I is done. The main problem with $\mathrm{V} 2 \mathrm{~V}$ is that it is time-consuming and might take a considerable amount of time to establish a connection and send the message. This is the major drawback of this algorithm.

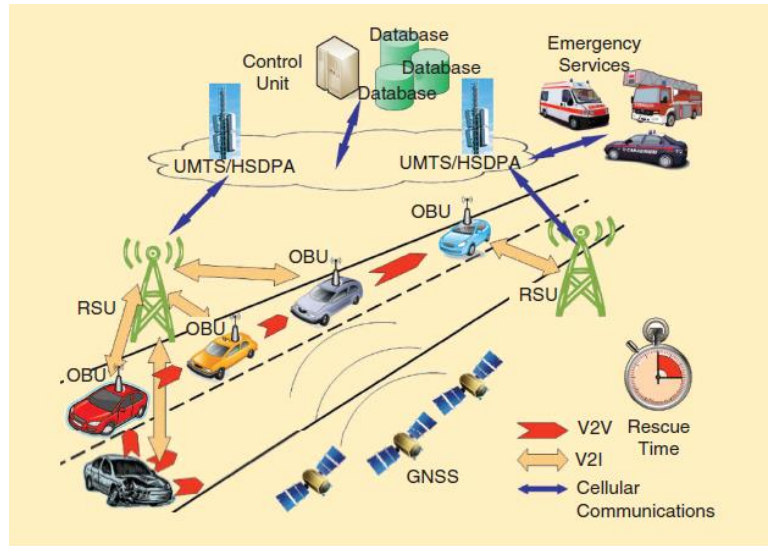

Fig.1 V2V and V2I communication

3. Vehicular Accidents Detection, SOS Alarm Triggering and Paging Algorithm

An effective algorithm for vehicular accidents detection, SOS alarm triggering and paging has been designed in this section and Fig 2 shows the basic design of the process. This process requires 4 important modules and is divided into 2 phases as- Triggering the Alarm and Sending Notification.

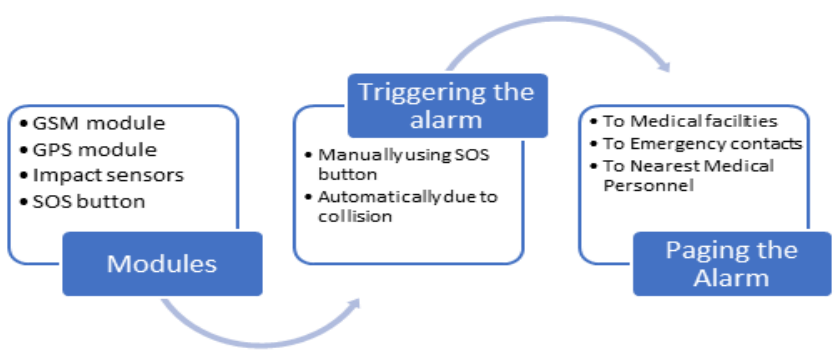

Fig. 2 Basic Design of the Algorithm

Both phases play an important role in this process. Both phases are explained as follows:

\subsection{Phase 1: Triggering of Alarm:}

This alarm in this algorithm can be triggered in two scenarios. In the first scenario, it can be manually triggered by the driver by pressing the SOS button that is present on the dashboard of the car. And in the second scenario, the alarm can be triggered if the impact sensor gives an electric impulse sensing a collision or an impact to the car.

First scenario:

In this case, the alarm can be triggered by the driver himself, if he is unable to drive the vehicle because of any medical emergency, SOS alarm can be triggered by the driver requesting for a medical emergency and as soon as this alarm is triggered, the vehicle comes to a halt with an alert sound to alarm the neighboring drivers about the halt and the situation to avoid further accidents. 
Second scenario:

In this case, the alarm is automatically triggered in the case of an accident in which the impact sensor senses the impact due to any collision and automatically sends a paging request. In both, the above cases mentioned how the alarm triggers vary, but the upcoming process is common.

\subsection{Phase 2: Paging the alarm:}

The next process is common in both scenarios. In the next step of this algorithm, sharing the location of the car is done. For that, contact information has to be set beforehand by the driver in case of an emergency. Apart from the emergency contact, this location is also shared with the nearest medical facility and also to National ambulance hotline number. The nearest medical facility can be located using a cellular network. The cellular network present in that cell locates the medical facility using the Base Station (BS). The request is sent to the nearest $\mathrm{BS}$, which forwards the request to the Mobile Service center (MSC). MSC then check for the medical facilities nearby and routes the paging message to that medical facility. The message includes the location of the car and also the car details, for easy identification of the vehicle.

Apart from the medical facility, these details are also sent to the nearby medical personnel, who is available to save the driver by providing the minimum first aid required to avoid cases like excessive blood loss or cases like heart stroke. These medical personnel can be located backend using the telecom customer data. This is done by predicting the customer's location depending on their previous location data, that is registered using either the Home Location Register (HLR) or Visitor Location Register (VLR) and updated in the MSC [8]. This updated location data is acquired for the prediction of medical personnel in that location [9]. Now, this data is used in sending a paging request to the medical personnel stating the emergency for a much quicker help before the medical services arrive to help the victim by providing the required first aid. In this way, the accident victims can be saved by providing them with immediate medical attention.

\section{Flow Chart}

The main algorithm can be divided into three different processes. Fig. 3 explains the first one, where the nearest medical facility can be located. Fig.4 explains the second process, where the nearest medical personnel is located by predicting the location of a mobile subscriber [9] using their previous location data. Finally, Fig.5 in which the processes of both Fig. 3 and Fig. 4 are used in the actual algorithm that is designed to notify the emergency services.

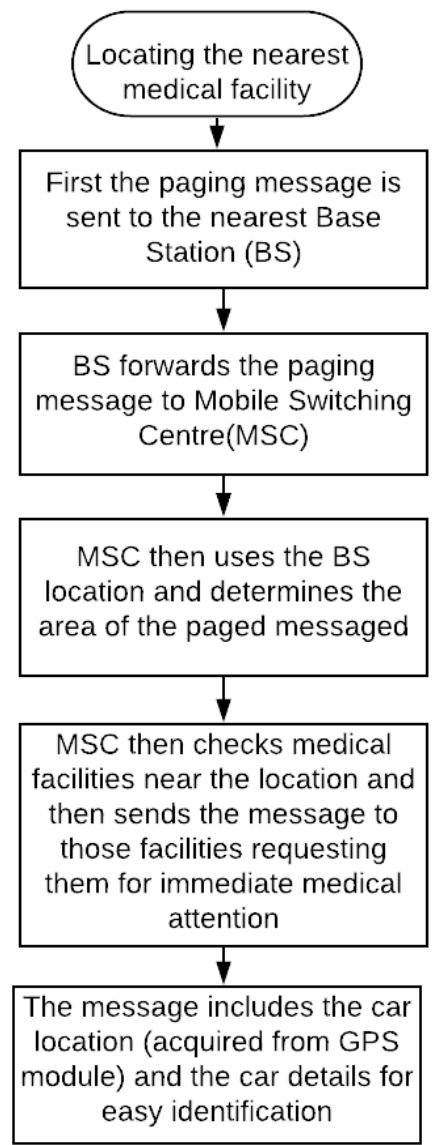

Fig.3 Process of Locating the nearest Medical facility

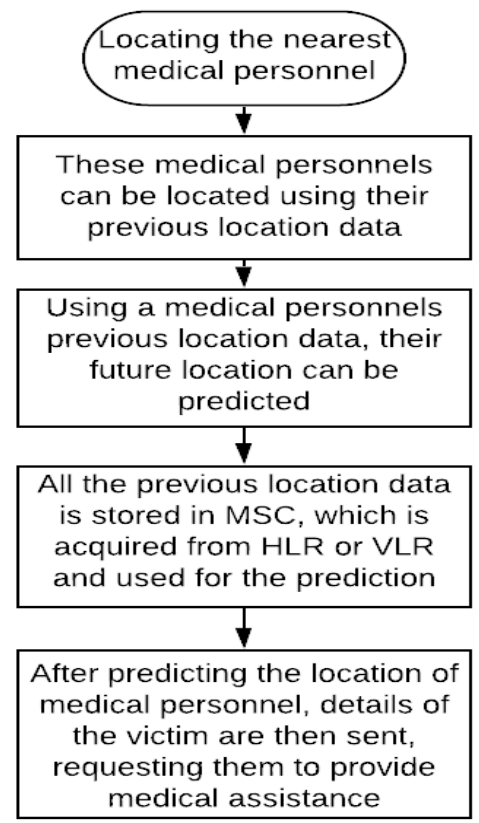

Fig.4 Process to Locate the nearest Medical Personnel 


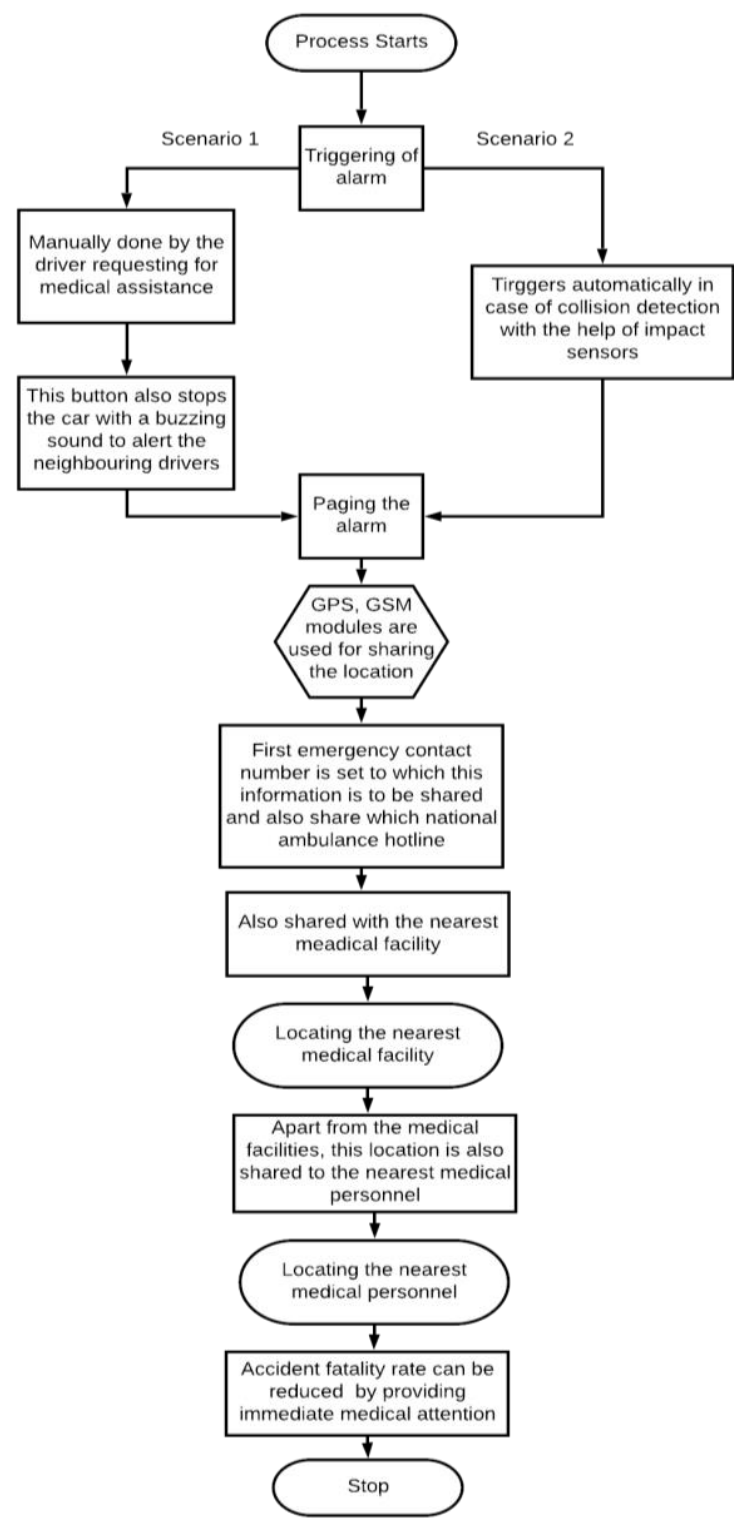

Fig.5 Flowchart of Proposed Algorithm

\section{Conclusion:}

In our day to day life, vehicular accidents are drastically increasing and the fatalities caused due to these accidents are also increasing. Vehicular accidents detection, SOS alarm triggering and paging algorithm helps in reducing these fatalities, that might be caused due to vehicular accidents. There are many existing algorithms existing, but a major drawback of those algorithms is, they are purely dependent on the mobile phones and if the mobile phone is not available at that point or the mobile phone gets damaged during the collision, the information fails to be notified to the medical facilities for immediate medical attention. So, to avoid this problem, Vehicular accidents detection, SOS alarm triggering and paging algorithm is designed which works independent of the mobile phone and is directly attached to the vehicle for better efficiency.

\section{References:}

[1] D. MOHAN, 'Road Accidents in India', IATSS Res., vol. 33, no. 1, pp. 75-79, 2009, doi: 10.1016/s03861112(14)60239-9.

[2] S. K. Singh, 'Road Traffic Accidents in India: Issues and Challenges', Transp. Res. Procedia, vol. 25, pp. 4708-4719, 2017, doi: 10.1016/j.trpro.2017.05.484.

[3] M. Syedul Amin, J. Jalil, and M. B. I. Reaz, 'Accident detection and reporting system using GPS, GPRS and GSM technology', 2012 Int. Conf. Informatics, Electron. Vision, ICIEV 2012, pp. 640-643, 2012, doi: 10.1109/ICIEV.2012.6317382.

[4] R. K. Megalingam, R. N. Nair, and S. M. Prakhya, 'Wireless vehicular accident detection and reporting system', ICMET 2010 - 2010 Int. Conf. Mech. Electr. Technol. Proc., no. Icmet, pp. 636-640, 2010, doi: 10.1109/ICMET.2010.5598437.

[5] E. Nasr, E. Kfoury, and D. Khoury, 'An IoT approach to vehicle accident detection, reporting, and navigation', 2016 IEEE Int. Multidiscip. Conf. Eng. Technol. IMCET 2016, pp. 231-236, 2016,

[6] A. Bin Faiz, A. Imteaj, and M. Chowdhury, 'Smart vehicle accident detection and alarming system using a smartphone', 1st Int. Conf. Comput. Inf. Eng. ICCIE 2015, pp. 66-69, 2016, doi: 10.1109/CCIE.2015.7399319.doi: 10.1109/IMCET.2016.7777457.

[7] M. Fogue, P. Garrido, F. J. Martinez, J. C. Cano, C. T. Calafate, and P. Manzoni, 'Automatic accident detection: Assistance through communication technologies and vehicles', IEEE Veh. Technol. Mag., vol. 7, no. 3, pp. 90100, 2012, doi: 10.1109/MVT.2012.2203877.

[8] Y. Wang et al., 'Regularity and conformity: Location prediction using heterogeneous mobility data', Proc. ACM SIGKDD Int. Conf. Knowl. Discov. Data Min., vol. 2015-August, pp. 1275-1284, 2015, doi: $10.1145 / 2783258.2783350$.

[9] T. H. N. Vu, K. H. Ryu, and N. Park, 'A method for predicting future location of mobile user for locationbased services system', Comput. Ind. Eng., vol. 57, no. 1, pp. 91-105, 2009, doi: 10.1016/j.cie.2008.07.009.

[10] H. M. Ali and Z. S. Alwan, 'Car Accident Detection and Notification System Using Smartphone', Int. J. Comput. Sci. Mob. Comput., vol. 44, no. 4, pp. 620-635, 2015.

[11] R. Dimple and B. S. Nanda, 'Design and implementation of smart black box system for gathering the safety information in vehicles', Int. J. Adv. Res. Ideas Innov. Technol., vol. 4, no. 3, pp. 1230-1233, 2018, [Online]. Available: www.ijariit.com. 